\title{
The Next Decade of Funding Opportunities for Mercury- Related Science and Mission Support
}

Lead Author: Kathleen E. Vander Kaaden, Jacobs/NASA Johnson Space Center (JSC), Kathleen.e.vanderkaaden@nasa.gov, (281) 244-5020

Co-authors:

Steven A. Hauck, II (Case Western Reserve University)

Carolyn M. Ernst (Johns Hopkins University Applied Physics Laboratory (JHU APL))

Ronald J. Vervack, Jr. (JHU APL)

Christian Klimczak (University of Georgia)

Catherine L. Johnson (University of British Columbia \& Planetary Science Institute)

Gina DiBraccio (NASA Goddard Space Flight Center (GSFC))

Ariel N. Deutsch (Brown University)

Gangkai Poh (NASA GSFC/Catholic University)

Suzanne Imber (University of Leicester)

Endorsers:

Maria E. Banks5 ${ }^{5}$ David T. Blewett4, Paul K. Byrne7, Nancy Chabot4, Mario D'Amore ${ }^{2}$, Ryan Dewey ${ }^{12}$, Sander Goossensi3, Cesare Grava10, James W. Head ${ }^{1}$, Chaneil James ${ }^{15}$, Lauren M. Jozwiak ${ }^{4}$, Scott King14, Rachel Klima4, Haje Korth ${ }^{4}$, Alice Lucchetti ${ }^{3}$, Francis M. McCubbin ${ }^{6}$, Maurizio Pajola ${ }^{3}$, Petr Pokorny ${ }^{11}$, Elizabeth Rampe ${ }^{6}$, James A Slavin ${ }^{12}$, Michelle Thompson ${ }^{9}$, Faith Vilas ${ }^{8}$

${ }^{1}$ Brown University, ${ }^{2}$ DLR German Aerospace Center, ${ }^{3}$ INAF-OAPD Astronomical Observatory of Padova, "The Johns Hopkins University Applied Physics Laboratory, ${ }^{5}$ NASA Goddard space Flight Center, ${ }^{6}$ NASA JSC, ${ }^{7}$ North Carolina State University, ${ }^{8}$ Planetary Science Institute, ${ }^{9}$ Purdue University, ${ }^{10}$ Southwest Research Institute, ${ }^{11}$ The Catholic University of America and Goddard space Flight Center, 12University of Michigan, ${ }^{13}$ Univ. of Maryland, Baltimore County \& NASA/GSFC, ${ }^{14}$ Virginia Tech, ${ }^{15} \mathrm{UCL}$ 
As an endmember for the study of rocky planet formation in our solar system, Mercury holds critical keys for unlocking mysteries of our inner solar system and beyond. Placing constraints on the formation, differentiation, and evolution of Mercury furthers comparative planetology studies that can yield transformative science relevant to the origin and composition of planetary building blocks in the solar system ${ }^{1}$. Continued exploration of the innermost planet is essential for addressing broad questions about the inner solar system and other rocky bodies that formed within close proximity to their host star. More specifically, the continued exploration of Mercury is crucial for increasing our understanding of the geochemistry, mineralogy, geology, tectonism, magnetism, and space environment of the planet itself ${ }^{2}$. With the immense success of NASA's MErcury Surface, Space ENvironment, GEochemistry, and Ranging (MESSENGER) mission, and the excitement surrounding the current ESA-JAXA BepiColombo mission, the Mercury-science community continues to grow. However, the strength of this community over the next decade will be dependent on funding opportunities that directly support Mercury-related science, as well as support for missions to the innermost planet.

\subsection{Introduction}

Exploration of Mercury (Figure 1) began as early as the seventeenth century, via ground-based telescopic observations - that continue today ${ }^{3}$. NASA's Mariner 10, which conducted three flybys of the planet in the 1970s and characterized just under 50\% of the planet's surface, was the first spacecraft to visit Mercury ${ }^{4}$. Our first global view of Mercury, however, was not obtained until NASA's MESSENGER mission, which orbited Mercury from 2011-2015. Although there are no current plans for the U.S. to lead a mission back to this enigmatic planet, the joint ESA-JAXA spacecraft (BepiColombo) was launched in 2018 and is en route to Mercury. BepiColombo will perform flybys of Venus (the first to occur in 2020) and Mercury before entering Mercury orbit in $2025^{5}$. MESSENGER provided unprecedented detail and data products that led to a revolution in Mercury science across the planetary sciences community. Within the U.S. specifically, MESSENGER's success cultivated the growth of a strong Mercury-science community.

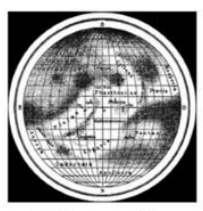

Telescopic Observations

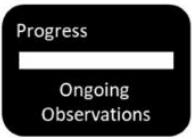

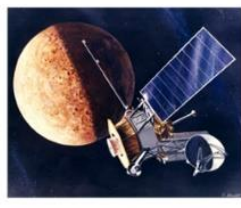

Flyby

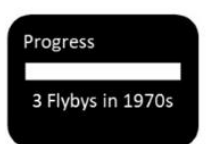

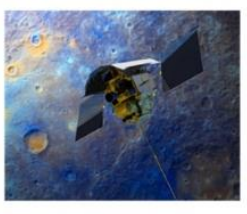

Orbiter

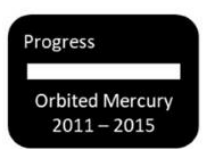

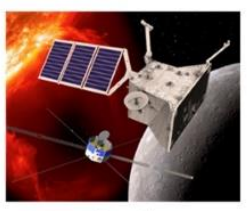

Dual Orbiter

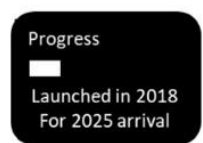

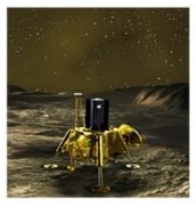

Lander

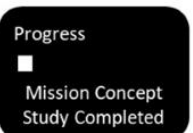

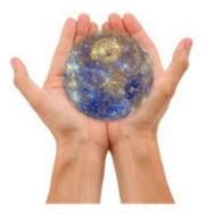

Sample Return

Progress I Brainstorming Underway

Figure 1. Visual representation of past, current, and future Mercury exploration efforts.

\footnotetext{
${ }^{1}$ Hauck et al. (2020) White Paper Submitted to the Decadal Survey

${ }^{2}$ https://link.springer.com/article/10.1007/s11214-019-0614-X

${ }^{3}$ https://airandspace.si.edu/exhibitions/exploring-the-planets/online/solar-system/mercury/earth-based-views.cfm

4 https://www.flickr.com/photos/128712737@N03/30668697263

5 https://www.esa.int/Enabling_Support/Operations/BepiColombo_operations
} 
Members of the Mercury-science community have recently completed a mission concept study in support of sending a lander to the surface as the next step in exploration by spacecraft ${ }^{6}$. In addition to such in situ measurements, there are critical science questions that can be only addressed via examination of a mercurian sample in Earth-based laboratories, i.e., presenting the additional need for a Mercury sample-return mission ${ }^{7}$. Mercury science continues to be motivated by a series of fundamental, interdisciplinary questions developed by the Mercury-science community $^{8}$. These questions include:

- How did Mercury form?

- How did Mercury differentiate and acquire its interior structure?

- What is the history of Mercury's magnetic field and its generation?

- How do Mercury's surface and interior reflect the evolution of the planet?

- What is the nature of the complex interactions among Mercury's external drivers and the planet's magnetosphere, exosphere, surface, and interior?

- What are the origin, history, and inventory of Mercury's volatiles?

To ensure that progress continues towards answering these overarching questions-vital for both understanding Mercury and the solar system and laying the groundwork for future spacecraft exploration - opportunities for research, analysis, and mission participation are essential. NASA created the Mercury Exploration Assessment Group (MExAG) in July 2020 in response to the strong support of the Mercury-science community across the planetary sciences, as well as a finding from the Planetary Science Advisory Committee (PAC) in February of 2018. The aim of this white paper, written by the membership of the MExAG Steering Committee, is to outline opportunities for supporting Mercury research and exploration activities for the science and exploration communities.

\subsection{Mercury-science Community Post-MESSENGER}

Although there are no current, U.S.-led missions planned to the Sun's closest planetary neighbor, the Mercury-science community, five years post-MESSENGER, is still vibrant. This energy is evident across many aspects of planetary science, for example:

- Mercury science continues to have dedicated oral and poster sessions at both planetaryfocused (e.g., the Lunar and Planetary Science Conference 2020 session "Mercury - The Little planet with a BIG personality!") and at non-planetary focused (e.g., American Geophysical Union 2020 session "Mercury: From MESSENGER to BepiColombo") science conferences;

- The Mercury Science e-mail listserv has 145 subscribers and the MExAG e-mail listserv has more than 50 subscribers as of September 2020;

- The Mercury 2018 meeting held in Columbia, MD, had 123 participants ${ }^{9}$ and the Mercury 2020 meeting (now Mercury $2021{ }^{10}$ due to travel restrictions associated with the pandemic) reached the max number of registrants at 121 and had seven other individuals on the waitlist;

\footnotetext{
${ }^{6}$ http://lib.jhuapl.edu/papers/mercury-lander/

${ }^{7}$ https://link.springer.com/article/10.1007/s11214-019-0614-X

${ }^{8}$ https://www.lpi.usra.edu/NASA-academies-resources/Mercury-high-priority-questions_final.pdf

${ }^{9}$ https://www.hou.usra.edu/meetings/mercury2018/Mercury2018_Report.pdf

${ }^{10}$ https://mercury2020.ias.u-psud.fr/main_1st.php
} 
- The high selection rates of the Discovery Data Analysis Program (DDAP), with regard to Mercury-related, MESSENGER-based, science (See Section 4.0);

- Almost 50 proposals, from U.S. investigators, were submitted to the 2019 ESA Announcement of Opportunity for Interdisciplinary Scientists (IDS) and Guest Investigators (GI) for the BepiColombo Mission ${ }^{11}$;

- Prior to the official designation of MExAG, nine members of the community formed an ad hoc committee to develop a report for NASA on high-priority science questions for the Decadal Survey ${ }^{12}$ and to draft the Terms of Reference for MExAG;

- More than 25 individuals applied for positions on the newly formed MExAG Steering Committee; and

- More than 40 white papers were submitted to the July 15, 2020 deadline for the Decadal Survey that fully focused on, or discussed applications to, future Mercury science and exploration.

As Mercury science continues to gain interest, it is vital that we maintain an active Mercuryscience community for several reasons:

1. NASA's investment in MESSENGER is best leveraged through continued research and analysis, which will help to solve ongoing (and new) Mercury-science questions and to further promote Mercury-related comparative planetology studies. The latter is especially important as Mercury's environment is the closest analog in our solar system to that of close-companion exoplanets. The continuation of Mercury science will enhance the scientific return of these data, make the most of the time and money investment that went into MESSENGER, establish the scientific motivation for future Mercury exploration, and maintain a work force prepared to execute future missions.

2. The successful completion of a Planetary Mission Concept Study for the 2023-2032 Decadal Survey has demonstrated that a New-Frontiers-class Mercury Lander is a viable option and would conduct transformative science on the surface of the innermost planet (Ernst et al. 202013).

3. NASA has an investment in the ongoing BepiColombo mission, namely in the NASAcontributed STROFIO (STart from a ROtating FIeld mass spectrOmeter) payload. The success of this activity and investment will be increased with an engaged and active Mercury-science community.

4.0 Selections of Mercury Science Proposals over the Past Six Years

Following the most recent PAC meeting (August 17 and 18, 2020), there is general concern from members across the field of planetary science regarding NASA's R\&A budget, the current pandemic's impact on current and future funding, and the availability of funding opportunities in the coming years. Not only are some typically annual programs not included in ROSES-20 or ROSES-21 -including Planetary Science and Technology from Analog Research (PSTAR) and Habitable Worlds - but also selection rates for other highly subscribed programs have dropped to

\footnotetext{
${ }^{11} \mathrm{https}$ ://sci.esa.int/web/bepicolombo/-/61341-announcement-of-opportunity-for-interdisciplinary-scientists-andguest-investigators-in-the-bepicolombo-mission

${ }^{12} \mathrm{https} / / /$ www.lpi.usra.edu/NASA-academies-resources/Mercury-high-priority-questions_final.pdf

${ }^{13}$ http://lib.jhuapl.edu/papers/mercury-lander/
} 
a concerning rate, e.g., Solar System Workings (SSW) selection rate has fallen from $22 \%$ in ROSES-18 to $11 \%$ in ROSES-19. The Mercury-science community, as well as other smaller scientific communities, can be disproportionately affected by low funding rates. Here, even a few non-selections of highly rated science proposals can lead to a significant loss of work force-for a body with limited spacecraft mission opportunities and long development and cruise times, a loss in the production of the future scientific workforce can be particularly harmful.

To understand past selection rates that may be indicative of future opportunities and to develop a better sense of the impact of these funding rates on the Mercury-science community, we analyzed the last six years (2014-2019) of selections made across five programs that have solicitations amenable to accommodating Mercury-related science: SSW, Planetary Data Archiving, Restoration, and Tools (PDART), Emerging Worlds (EW), Solar System Observations (SSO), and DDAP. Using the publicly available NASA Solicitation and Proposal Integrated Review and Evaluation System (NSPIRES) selection documents, we examined the total number of proposals submitted, the total number of proposals selected, as well as the total number of proposals selected that would conduct research related to Mercury. As the latter determinations are quite subjective, we were extremely lenient in our determination of what selections were considered Mercury related. For example, a 2019 SSW selection entitled "Dynamics and Evolution of Circumsolar Dust Rings" was counted as "research related to Mercury" because the abstract stated, "Our team proposes to analyze the dynamics and evolution of circumsolar dust rings in the inner solar system from Mercury to Jupiter" (Petr Pokorny/The Catholic University of America). Although this research did not seem to be studying Mercury specifically, it is looking at the environment around Mercury which, to some extent, affects the planet itself. Therefore, the data presented here should be viewed as maximum values. The results of these analyses are shown in Figure 2 and Table 1.

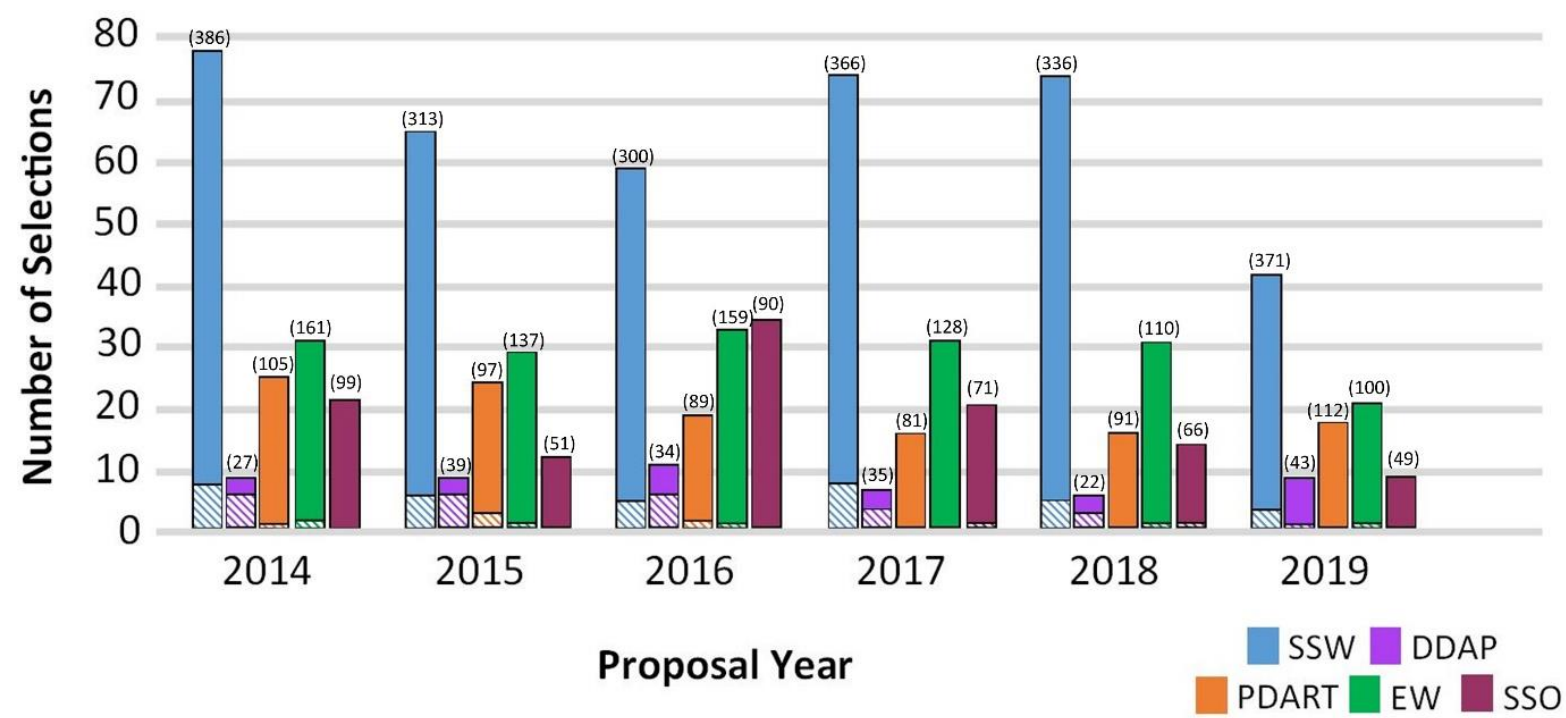

(Total number of proposals submitted) $\square$ Total Selections $\mathbb{N}$ Total Mercury-Related Selections

Figure 2. Number of funded proposals for Mercury related work, by program from 2014-2019 for SSW, DDAP, PDART, EW, and SSO. In the event a color figure is not available, each year is ordered SSW, DDAP, PDART, EW, then SSO from left to right.

Although there were no NSPIRES selection documents available for SSO for 2018, the data were provided to us by a NASA program officer. Figure 2 displays the total number of funded 
proposals by year for each of the five programs (solid bar), the total number of funded proposals by year for each program which were determined to be Mercury-related (diagonal shading bar), and the total number of proposals submitted to each program by year (in parentheses). Prior to interpreting the data shown in Figure 2, there are important caveats that must be stated:

1. We do not have data to show the total number of Mercury related proposals that were submitted to each of these programs.

2. We assume, given the vibrant nature of our Mercury-science community, and the high percentage of Mercury-related proposals selected across the DDAP program, that there are a significant number of Mercury-related science proposals being submitted but not selected. This is also supported by the more than 40 proposals submitted to the 2019 BepiColombo IDS and GI opportunity.

3. There is crucial information lacking in our current data that is imperative to interpreting the results. For example, there is a decline in overall DDAP selection rates from 2014 (9 selected out of 27 submissions) to 2019 (9 selected out of 43 submissions). There is also a decline in the rate of Mercury-related DDAP proposals selected from 2014 (6 out of 27) to 2019 ( 1 out of 43). This could be caused by numerous variables, such as: fewer Mercuryrelated proposals submitted to DDAP-19 than DDAP-14 because of higher rates of funding for Mercury-related science in previous years; the addition of new datasets over the course of these six years changing the funding scheme of the program; a lack of funding for the program decreasing the selection rate in recent years, and so on.

Table 1. Number of Mercury-related proposals selected by program for given years.

\begin{tabular}{|c|c|c|c|c|c|c|}
\hline & $\mathbf{2 0 1 4}$ & $\mathbf{2 0 1 5}$ & $\mathbf{2 0 1 6}$ & $\mathbf{2 0 1 7}$ & $\mathbf{2 0 1 8}$ & $\mathbf{2 0 1 9}$ \\
\hline SSW & 8 & 6 & 5 & 8 & 5 & 4 \\
\hline DDAP & 6 & 6 & 6 & 4 & 3 & 1 \\
\hline PDART & 1 & 3 & 2 & 0 & 0 & 0 \\
\hline EW & 2 & 1 & 1 & 0 & 1 & 1 \\
\hline SSO & 0 & 0 & 0 & 1 & 1 & 0 \\
\hline
\end{tabular}

The average overall selection rates (defined as the total number of selected proposals divided by the total number of proposals submitted for a given program) for these five programs over the last six years is $19 \%$ for SSW, 26\% for DDAP, 21\% for PDART, 22\% for EW, and 25\% for SSO. The average Mercury-related selection rates (defined here as the number of Mercury-related proposals selected divided by the total number of proposals submitted) for these five programs over the last six years is $2 \%$ for SSW, $14 \%$ for DDAP, $1 \%$ for PDART, $1 \%$ for EW, and <1\% for SSO. It is important to note (see Table 1) that there were no Mercury-related proposals selected in PDART from 2017-2019, in EW in 2017, and in SSO from 2014-2016 and again in 2019 (Table 1). A single Mercury-related proposal was selected in DDAP in 2019, in PDART in 2014, in EW in 2015, 2016, 2018, and 2019, and in SSO in 2017 and 2018. When comparing the number of Mercury-related proposals funded five and six years ago (2014/2015) with the more recent number of selections over the last two years (2018/2019), there is a general decrease in the number of Mercury-related proposals being funded across these five programs, just at the time the in-depth work connecting laboratory, theoretical, and interdisciplinary (and inter-planetary body) research stemming from the MESSENGER mission and in preparation for BepiColombo (including the NASA funded STROFIO instrument) should be hitting its stride.

Out of these five programs, the DDAP selection rates are the most favorable for Mercury- 
related research. From 2014-2019, the average selection rate for Mercury-related science in DDAP was $14 \%$. This selection rate is enabling for maintaining a strong Mercury-science community. However, two trends are concerning. First, there is a clear decline in DDAP selections over the last six years from a 22\% selection of Mercury-related science in 2014 to a $2 \%$ selection of Mercury-related science in 2019. This is particularly alarming for the Mercury-science community because in 2014, there was a 33\% overall selection rate (27 DDAP proposals submitted and 9 selected, 6 of which were Mercury-related) and in 2019 there was a $21 \%$ overall selection rate (43 DDAP proposals submitted and 9 selected, but only one was Mercury-related). Therefore, although the number of proposals submitted significantly increased from 2014 to 2019, the same number of proposals were selected, and the number of Mercury-related proposals drastically decreased. Second, the objective of the DDAP program is to enhance the scientific return of Discovery missions, which currently only includes MESSENGER for Mercury-centric research. Given the limited missions to Mercury, there are limited datasets that fall into this category and therefore limited opportunities to support Mercury scientists. Because of this, anyone in the Mercury-science community proposing to conduct fundamental research that does not specifically utilize MESSENGER data must compete in the other core programs where the selections are highly competitive, which can severely impact smaller scientific communities.

Aside from typical yearly ROSES solicitations, there was an opportunity in 2019 for US-based scientists to apply to an ESA-run Announcement of Opportunity for Interdisciplinary Scientists (IDSs) and Guest Investigators (GIs) for the BepiColombo mission (with selected U.S. scientists funded by NASA). The intent for the IDS positions were for scientists to focus on broad themes (e.g., mineralogy, exosphere) that were not specific-instrument-dependent, to facilitate crossdisciplinary science investigations. The GI positions, on the other hand, were intended for individual scientists to use specific instrument data in combination with various techniques (e.g., ground-based observations, laboratory measurements). There were 19 proposals submitted for the IDS positions and 28 proposals submitted for the GI positions from U.S.-based investigators (30 IDS and 37 GI proposals were submitted in total). Although the announcement of opportunity stated "the aim of this AO is to appoint up to six IDSs and up to eleven GIs for the BepiColombo mission", selections included a single US-based IDS proposal (5\% selection rate) and only two US-based GI proposals (7\% selection rate). Furthermore, one of the U.S.-based selections that was made will utilize Venus fly-by data and was not a Mercury-related selection.

The results of these data shed light on a few important conclusions: 1) Mercury does not benefit from a direct line of funding in the Planetary Science Division that the study of other planetary bodies have access to (i.e., Mars and MDAP/the Mars Exploration Program, Moon and LDAP, astrobiology and Habitable Worlds/Exobiology) resulting in Mercury-related proposals being submitted to large core programs; 2) the opportunities that do exist are subject to extremely competitive selection rates; and 3) there is currently no NASA program that specifically calls for use of BepiColombo data, despite the NASA contribution to this mission.

\section{0 Mission Support}

To date there have only been two missions that have explored Mercury: Mariner 10 and MESSENGER. There are currently no NASA-funded missions planned to continue the exploration of this end-member planet. Fortunately, the ESA-JAXA dual orbiter spacecraft BepiColombo is currently on its way to the innermost planet. It is anticipated that future exploration efforts of 
Mercury by BepiColombo, landed science, and eventual sample return will continue to revolutionize our understanding of not only Mercury, but our solar system as a whole. It is therefore critical that NASA continue to support missions to study the innermost planet over and beyond the next decade.

There is currently an opportunity to leverage data that are obtained from the ESA-JAXA BepiColombo mission to continue our exploration of the Sun's closest planetary neighbor. We urge NASA to consider supporting analysis of data from international spaceflight missions, e.g., within the DDAP or in another mission-specific program (the Rosetta Data Analysis Program, for example, is a relevant and recent precedent for this kind of data analysis program), especially when such data are highly complementary to recent (i.e., MESSENGER) and current (i.e., STROFIO) NASA investments. Furthermore, the BepiColombo flybys of Venus will collect new data for another planetary body in the inner solar system that is severely lacking recent data collection from NASA missions. It would thus be extremely advantageous for members of multiple planetary science communities if BepiColombo data were either included in DDAP for future solicitations or if an additional call were created for analysis of data from BepiColombo.

6.0 The Importance of Diversity, Equity, Inclusion, and Accessibility in Planetary Science

Team dynamics, diversity, equity, inclusion, and accessibility (DEIA) are critical to the success of planetary sciences. Members of both the Equity, Diversity, and Inclusion Working Group (EDIWG) and the planetary sciences community have drafted many white papers submitted to this Decadal Survey call discussing such topics. It is vital that the planetary science community fosters an interdisciplinary, diverse, equitable, inclusive, and accessible environment over the next decade to ensure the success and sustainability of our field. We strongly urge members of the committees supporting the drafting of the Decadal Survey to read each of these papers and integrate their various recommendations into the final survey document, as appropriate.

\subsection{Recommendations}

The sustainability of the Mercury-science community - and the work force that leverages past NASA investments and enables future missions - is dependent on broadening and developing funding opportunities for Mercury-related science and exploration. In addition, it is critical that NASA fosters further collaborations and opportunities with ESA and JAXA to afford U.S.-based scientists continued funded opportunities to work on BepiColombo mission data. Through the expansion of these activities, the Mercury-science community can have the ability to thrive over the next decade. Below is a list of recommendations that should be implemented over the next decade to support members of the Mercury-science community (in no particular order):

- Increase funding selection rates for R\&A programs.

- Include funding opportunities for education and public outreach of Mercury science.

- Support future opportunities for additional IDS and GI positions on BepiColombo.

- Expand the DDAP call to include data from BepiColombo or create an additional call for a BepiColombo Data Analysis Program.

- Add NASA-led missions to Mercury to the New Frontiers call (e.g., a Mercury Lander).

- Consider the critical role of team dynamics, equity, diversity, inclusion, and accessibility not just in the continued exploration of Mercury, but across the field of planetary science. 